\title{
Matroid Polytopes and Their Volumes
}

\author{
Federico Ardila 1 , Carolina Benedetti ${ }^{2}$ and Jeffrey Doker ${ }^{3}$
}

${ }^{1}$ San Francisco, CA, USA, federico@math.sfsu.edu.

${ }^{2}$ Universidad de Los Andes, Bogotá, Colombia, c.benedetti92@uniandes.edu.co.

${ }^{3}$ University of California, Berkeley, Berkeley, CA, USA, doker@math.berkeley.edu.

\begin{abstract}
We express the matroid polytope $P_{M}$ of a matroid $M$ as a signed Minkowski sum of simplices, and obtain a formula for the volume of $P_{M}$. This gives a combinatorial expression for the degree of an arbitrary torus orbit closure in the Grassmannian $G r_{k, n}$. We then derive analogous results for the independent set polytope and the associated flag matroid polytope of $M$. Our proofs are based on a natural extension of Postnikov's theory of generalized permutohedra.

Résumé. On exprime le polytope matroïde $P_{M}$ d'un matroïde $M$ comme somme signée de Minkowski de simplices, et on obtient une formule pour le volume de $P_{M}$. Ceci donne une expression combinatoire pour le degré d'une clôture d'orbite de tore dans la Grassmannienne $G r_{k, n}$. Ensuite, on deduit des résultats analogues pour le polytope ensemble indépendant et pour le polytope matroïde drapeau associé a $M$. Nos preuves sont fondées sur une extension naturelle de la théorie de Postnikov de permutoèdres généralisés.
\end{abstract}

Keywords: Matroid; generalized permutohedron; matroid polytope; Minkowski sum; mixed volume; flag matroid.

\footnotetext{
${ }^{\dagger}$ NSF grant DMS-0801075

$\ddagger$ Proyecto Semilla of the Universidad de Los Andes

1365-8050 @ 2009 Discrete Mathematics and Theoretical Computer Science (DMTCS), Nancy, France
} 


\section{Introduction}

The theory of matroids can be approached from many different points of view; a matroid can be defined as a simplicial complex of independent sets, a lattice of flats, a closure relation, etc. A relatively new point of view is the study of matroid polytopes, which in some sense are the natural combinatorial incarnations of matroids in algebraic geometry and optimization. Our paper is a contribution in this direction.

We begin with the observation that matroid polytopes are members of the family of generalized permutohedra (14). With some modifications of Postnikov's beautiful theory, we express the matroid polytope $P_{M}$ as a signed Minkowski sum of simplices, and use that to give a formula for its volume $\operatorname{Vol}\left(P_{M}\right)$. This is done in Theorems 2.5 and 3.3 . Our answers are expressed in terms of the beta invariants of the contractions of $M$.

Formulas for $\operatorname{Vol}\left(P_{M}\right)$ were given in very special cases by Stanley (17) and Lam and Postnikov (11), and a polynomial time algorithm for finding $\operatorname{Vol}\left(P_{M}\right)$ was constructed by de Loera et. al. (6). One motivation for this computation is the following. The closure of the torus orbit of a point $p$ in the Grassmannian $G r_{k, n}$ is a toric variety $X_{p}$, whose degree is the volume of the matroid polytope $P_{M_{p}}$ associated to $p$. Our formula allows us to compute the degree of $X_{p}$ combinatorially.

One can naturally associate two other polytopes to a matroid $M$ : its independent set polytope and its associated flag matroid polytope. By a further extension of Postnikov's theory, we also write these polytopes as signed Minkowski sums of simplices and give formulas for their volumes. This is the content of Sections 4 and 5 .

Throughout the paper we assume familiarity with the basic concepts of matroid theory; for further information we refer the reader to (13).

\section{Matroid Polytopes are Generalized Permutohedra}

A generalized permutohedron is a polytope whose inequality description is of the following form:

$$
P_{n}\left(\left\{z_{I}\right\}\right)=\left\{\left(t_{1}, \ldots, t_{n}\right) \in \mathbb{R}^{n}: \sum_{i=1}^{n} t_{i}=z_{[n]}, \sum_{i \in I} t_{i} \geq z_{I} \text { for all } I \subseteq[n]\right\}
$$

where $z_{I}$ is a real number for each $I \subseteq[n]:=\{1, \ldots, n\}$, and $z_{\emptyset}=0$. Different choices of $z_{I}$ can give the same generalized permutohedron: if one of the inequalities does not define a face of $P_{n}\left(\left\{z_{I}\right\}\right)$, then we can increase the value of the corresponding $z_{I}$ without altering the polytope. When we write $P_{n}\left(\left\{z_{I}\right\}\right)$, we will always assume that the $z_{I} \mathrm{~s}$ are all chosen minimally; i.e., that all the defining inequalities are tight.

The Minkowski sum of two polytopes $P$ and $Q$ in $\mathbb{R}^{n}$ is defined to be $P+Q=\{p+q: p \in P, q \in Q\}$. We say that the Minkowski difference of $P$ and $Q$ is $P-Q=R$ if $P=Q+R\left[{ }^{[\text {(i) }}\right.$ The following lemma shows that generalized permutohedra behave nicely with respect to Minkowski sums.

Lemma 2.1 $P_{n}\left(\left\{z_{I}\right\}\right)+P_{n}\left(\left\{z_{I}^{\prime}\right\}\right)=P_{n}\left(\left\{z_{I}+z_{I}^{\prime}\right\}\right)$.

\footnotetext{
(i) We will only consider Minkowski differences $P-Q$ such that $Q$ is a Minkowski summand of $P$. More generally, the Minkowski difference of two arbitrary polytopes $P$ and $Q$ in $\mathbb{R}^{n}$ is defined to be $P-Q=\left\{r \in \mathbb{R}^{n} \mid r+Q \subseteq P\right\}$ (14). It is easy to check that $(Q+R)-Q=R$, so the two definitions agree in the cases that interest us. In this paper, a signed Minkowski sum equality such as $P-Q+R-S=T$ should be interpreted as $P+R=Q+S+T$.
} 
Let $\Delta$ be the standard unit $(n-1)$-simplex

$$
\begin{aligned}
\Delta & =\left\{\left(t_{1}, \ldots, t_{n}\right) \in \mathbb{R}^{n}: \sum_{i=1}^{n} t_{i}=1, t_{i} \geq 0 \text { for all } 1 \leq i \leq n\right\} \\
& =\operatorname{conv}\left\{e_{1}, \ldots, e_{n}\right\}
\end{aligned}
$$

where $e_{i}=(0, \ldots, 0,1,0, \ldots, 0)$ with a 1 in its $i$ th coordinate. As $J$ ranges over the subsets of $[n]$, let $\Delta_{J}$ be the face of the simplex $\Delta$ defined by

$$
\Delta_{J}=\operatorname{conv}\left\{e_{i}: i \in J\right\}=P_{n}\left(\left\{z(J)_{I}\right\}\right)
$$

where $z(J)_{I}=1$ if $I \supseteq J$ and $z(J)_{I}=0$ otherwise. Lemma 2.1 gives the following proposition.

The next two propositions are due to Postnikov in the case $y_{I} \geq 0$.

Proposition 2.2 (14. Proposition 6.3) For any $y_{I} \geq 0$, the Minkowski sum $\sum y_{I} \Delta_{I}$ of dilations of faces of the standard $(n-1)$-simplex is a generalized permutohedron. We can write

$$
\sum_{A \subseteq E} y_{I} \Delta_{I}=P_{n}\left(\left\{z_{I}\right\}\right)
$$

where $z_{I}=\sum_{J \subseteq I} y_{J}$ for each $I \subseteq[n]$.

Proposition 2.3 Every generalized permutohedron $P_{n}\left(\left\{z_{I}\right\}\right)$ can be written uniquely as a signed Minkowski sum of simplices, as

$$
P_{n}\left(\left\{z_{I}\right\}\right)=\sum_{I \subseteq[n]} y_{I} \Delta_{I}
$$

where $y_{I}=\sum_{J \subseteq I}(-1)^{|I|-|J|} z_{J}$ for each $I \subseteq[n]$

Proof: First we need to separate the right hand side into its positive and negative parts. By Proposition 2.2 .

$$
\sum_{I \subseteq[n]: y_{I}<0}\left(-y_{I}\right) \Delta_{I}=P_{n}\left(\left\{z_{I}^{-}\right\}\right) \text {and } \sum_{I \subseteq[n]: y_{I} \geq 0} y_{I} \Delta_{I}=P_{n}\left(\left\{z_{I}^{+}\right\}\right)
$$

where $z_{I}^{-}=\sum_{J \subseteq I: y_{J}<0}\left(-y_{J}\right)$ and $z_{I}^{+}=\sum_{J \subseteq I: y_{J} \geq 0} y_{J}$. Now $z_{I}+z_{I}^{-}=z_{I}^{+}$gives

$$
P_{n}\left(\left\{z_{I}\right\}\right)+\sum_{I \subseteq[n]: y_{I}<0}\left(-y_{I}\right) \Delta_{I}=\sum_{I \subseteq[n]: y_{I} \geq 0} y_{I} \Delta_{I}
$$

as desired. Uniqueness is clear.

Let $M$ be a matroid of rank $r$ on the set $E$. The matroid polytope of $M$ is the polytope $P_{M}$ in $\mathbb{R}^{E}$ whose vertices are the indicator vectors of the bases of $M$. The known description of the polytope $P_{M}$ by inequalities makes it apparent that it is a generalized permutohedron:

Proposition 2.4 (19) The matroid polytope of a matroid $M$ on $E$ with rank function $r$ is $P_{M}=P_{E}(\{r-$ $\left.r(E-I)\}_{I \subseteq E}\right)$. 
The beta invariant (5) of $M$ is a non-negative integer given by

$$
\beta(M)=(-1)^{r(M)} \sum_{X \subseteq E}(-1)^{|X|} r(X)
$$

which stores significant information about $M$; for example, $\beta(M)=0$ if and only if $M$ is disconnected and $\beta(M)=1$ if and only if $M$ is series-parallel. If

$$
T_{M}(x, y)=\sum_{A \subseteq E}(x-1)^{r(E)-r(A)}(y-1)^{|A|-r(A)}=\sum_{i, j} b_{i j} x^{i} y^{j}
$$

is the Tutte polynomial (20) of $M$, then $\beta(M)=b_{10}=b_{01}$ for $|E| \geq 2$.

Our next results are more elegantly stated in terms of the signed beta invariant of $M$, which we define to be

$$
\widetilde{\beta}(M)=(-1)^{r(M)+1} \beta(M) .
$$

Theorem 2.5 Let $M$ be a matroid of rank $r$ on $E$ and let $P_{M}$ be its matroid polytope. Then

$$
P_{M}=\sum_{A \subseteq E} \widetilde{\beta}(M / A) \Delta_{E-A} .
$$

Proof: By Propositions 2.3 and 2.4. $P_{M}=\sum_{I \subseteq E} y_{I} \Delta_{I}$ where

$$
\begin{aligned}
y_{I} & =\sum_{J \subseteq I}(-1)^{|I|-|J|}(r-r(E-J))=-\sum_{J \subseteq I}(-1)^{|I|-|J|} r(E-J) \\
& =-\sum_{E-J \supseteq E-I}(-1)^{|E-J|-|E-I|}(r(E-J)-r(E-I)) \\
& =-\sum_{X \subseteq I}(-1)^{|X|}(r(E-I \cup X)-r(E-I)) \\
& =-\sum_{X \subseteq I}(-1)^{|X|} r_{M /(E-I)}(X)=\widetilde{\beta}(M /(E-I))
\end{aligned}
$$

as desired.

Example 2.6 Let $M$ be the matroid on $E=[4]$ with bases $\{12,13,14,23,24\}$; its matroid polytope is a square pyramid. Theorem 2.5 gives $P_{M}=\Delta_{234}+\Delta_{134}+\Delta_{12}-\Delta_{1234}$, as illustrated in Figure 1 . The dotted lines in the polytope $\Delta_{234}+\Delta_{134}+\Delta_{12}$ are an aid to visualize the Minkowski difference.

One way of visualizing the Minkowski sum of two polytopes $P$ and $Q$ is by grabbing a vertex $v$ of $Q$ and then using it to "slide" $Q$ around in space, making sure that $v$ never leaves $P$. The region that $Q$ sweeps along the way is $P+Q$. Similarly, the Minkowski difference $P-R$ can be visualized by picking a vertex $v$ of $R$ and then "sliding" $R$ around in space, this time making sure that no point in $R$ ever leaves $P$. The region that $v$ sweeps along the way is $P-R$. This may be helpful in understanding Figure 1

Some remarks about Theorem 2.5 are in order. 


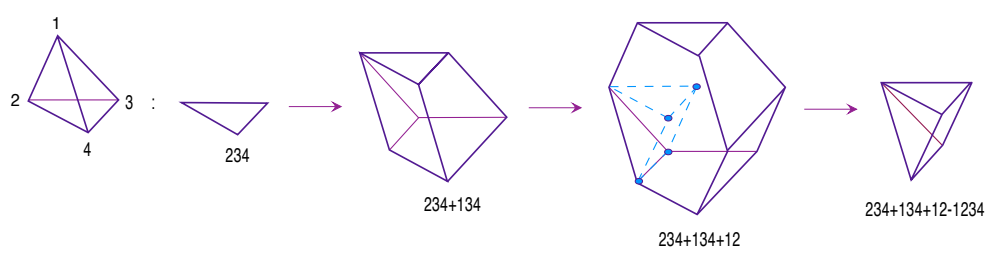

Fig. 1: A matroid polytope as a signed Minkowski sum of simplices.

- Generally most terms in the sum of Theorem 2.5 are zero. The nonzero terms correspond to the coconnected flats $A$, which we define to be the sets $A$ such that $M / A$ is connected. These are indeed flats, since contracting by them must produce a loopless matroid.

- A matroid and its dual have congruent matroid polytopes, and Theorem 2.5 gives different formulas for them. For example $P_{U_{1,3}}=\Delta_{123}$ while

$$
P_{U_{2,3}}=\Delta_{12}+\Delta_{23}+\Delta_{13}-\Delta_{123} \text {. }
$$

- The study of the subdivisions of a matroid polytope into smaller matroid polytopes, originally considered by Lafforgue (10), has recently received significant attention (1, 2, 7, 15). Speyer conjectured (15) that the subdivisions consisting of series-parallel matroids have the largest number of faces in each dimension and proved this (16) for a large and important family of subdivisions: those which arise from a tropical linear space. The important role played by series-parallel matroids is still somewhat mysterious. Theorem 2.5 characterizes series-parallel matroids as those whose matroid polytope has no repeated Minkowski summands. It would be interesting to connect this characterization to matroid subdivisions; this may require extending the theory of mixed subdivisions to signed Minkowski sums.

- Theorem 2.5 provides a geometric interpretation for the beta invariant of a matroid $M$ in terms of the matroid polytope $P_{M}$. In Section 5 we see how to extend this to certain families of Coxeter matroids. This is a promising point of view towards the notable open problem (4, Problem 6.16.6) of defining useful enumerative invariants of a Coxeter matroid.

\section{The Volume of a Matroid Polytope}

Our next goal is to present an explicit combinatorial formula for the volume of an arbitrary matroid polytope. Formulas have been given for very special families of matroids by Stanley (17) and Lam and Postnikov (11). Additionally, a polynomial time algorithm for computing the volume of an arbitrary matroid polytope was recently discovered by de Loera et. al. (6). Let us say some words about the motivation for this question.

Consider the Grassmannian manifold $G r_{k, n}$ of $k$-dimensional subspaces in $\mathbb{C}^{n}$. Such a subspace can be represented as the rowspace of a $k \times n$ matrix $A$ of rank $k$, modulo the left action of $G L_{k}$ which does not change the row space. The $\left(\begin{array}{l}n \\ k\end{array}\right)$ maximal minors of this matrix are the Plücker coordinates of the subspace, and they give an embedding of $G r_{k, n}$ as a projective algebraic variety in $\left.\mathbb{C P}^{(} \begin{array}{l}n \\ k\end{array}\right)-1$. 
Each point $p$ in $G r_{k, n}$ gives rise to a matroid $M_{p}$ whose bases are the $k$-subsets of $n$ where the Plücker coordinate of $p$ is not zero. Gelfand, Goresky, MacPherson, and Serganova (9) first considered the stratification of $G r_{k, n}$ into matroid strata, which consist of the points corresponding to a fixed matroid.

The torus $\mathbb{T}=\left(\mathbb{C}^{*}\right)^{n}$ acts on $\mathbb{C}^{n}$ by $\left(t_{1}, \ldots, t_{n}\right) \cdot\left(x_{1}, \ldots, x_{n}\right)=\left(t_{1} x_{1}, \ldots, t_{n} x_{n}\right)$ for $t_{i} \neq 0$; this action extends to an action of $\mathbb{T}$ on $G r_{k, n}$. For a point $p \in G r_{k, n}$, the closure of the torus orbit $X_{p}=\overline{\mathbb{T} \cdot p}$ is a toric variety which only depends on the matroid $M_{p}$ of $p$, and the polytope corresponding to $X_{p}$ under the moment map is the matroid polytope of $M_{p}(9)$. Under these circumstances it is known (8) that the volume of the matroid polytope $M_{p}$ equals the degree of the toric variety $X_{p}$ as a projective subvariety of $\mathbb{C P}\left(\begin{array}{l}n \\ k\end{array}\right)-1$ :

$$
\operatorname{Vol} P_{M_{p}}=\operatorname{deg} X_{p} \text {. }
$$

Therefore, by finding the volume of an arbitrary matroid polytope, one obtains a formula for the degree of the toric varieties arising from arbitrary torus orbits in the Grassmannian.

To prove our formula for the volume of a matroid polytope, we first recall the notion of the mixed volume $\operatorname{Vol}\left(P_{1}, \ldots, P_{n}\right)$ of $n$ (possibly repeated) polytopes $P_{1}, \ldots, P_{n}$ in $\mathbb{R}^{n}$. All volumes in this section are normalized with respect to the lattice generated by $e_{1}-e_{2}, \ldots, e_{n-1}-e_{n}$ where our polytopes live; so the standard simplex $\Delta$ has volume $1 /(n-1)$ !.

Proposition 3.1 (12) Let $n$ be a fixed positive integer. There exists a unique function $\operatorname{Vol}\left(P_{1}, \ldots, P_{n}\right)$ defined on $n$-tuples of polytopes in $\mathbb{R}^{n}$, called the mixed volume of $P_{1}, \ldots, P_{n}$, such that, for any collection of polytopes $Q_{1}, \ldots, Q_{m}$ in $\mathbb{R}^{n}$ and any nonnegative real numbers $y_{1}, \ldots, y_{m}$, the volume of the Minkowski sum $y_{1} Q_{1}+\cdots+y_{m} Q_{m}$ is the polynomial in $y_{1}, \ldots, y_{m}$ given by

$$
\operatorname{Vol}\left(y_{1} Q_{1}+\cdots+y_{m} Q_{m}\right)=\sum_{i_{1}, \ldots, i_{n}} \operatorname{Vol}\left(Q_{i_{1}}, \ldots, Q_{i_{n}}\right) y_{i_{1}} \cdots y_{i_{n}}
$$

where the sum is over all ordered $n$-tuples $\left(i_{1}, \ldots, i_{n}\right)$ with $1 \leq i_{r} \leq m$.

Proposition 3.1 still holds if some of the $y_{i}$ s are negative as long as the expression $y_{1} Q_{1}+\cdots+y_{m} Q_{m}$ still makes sense, as stated in the following Proposition.

Proposition 3.2 If $P=y_{1} Q_{1}+\cdots+y_{m} Q_{m}$ is a signed Minkowski sum of polytopes in $\mathbb{R}^{n}$, then

$$
\operatorname{Vol}\left(y_{1} Q_{1}+\cdots+y_{m} Q_{m}\right)=\sum_{i_{1}, \ldots, i_{n}} \operatorname{Vol}\left(Q_{i_{1}}, \ldots, Q_{i_{n}}\right) y_{i_{1}} \cdots y_{i_{n}}
$$

where the sum is over all ordered $n$-tuples $\left(i_{1}, \ldots, i_{n}\right)$ with $1 \leq i_{r} \leq m$.

Proof: We first show that

$$
\operatorname{Vol}(A-B)=\sum_{k=0}^{n}(-1)^{k}\left(\begin{array}{l}
n \\
k
\end{array}\right) \operatorname{Vol}(A, \ldots, A, B, \ldots, B)
$$

when $B$ is a Minkowski summand of $A$ in $\mathbb{R}^{n}$. Let $A-B=C$. By Proposition 3.1 , for $t \geq 0$ we have that

$$
\operatorname{Vol}(C+t B)=\sum_{k=0}^{n}\left(\begin{array}{l}
n \\
k
\end{array}\right) \operatorname{Vol}(C, \ldots, C, B \ldots, B) t^{k}=: f(t)
$$


and we are interested in computing $\operatorname{Vol}(C)=f(0)$. Invoking Proposition 3.1 again, for $t \geq 0$ we have that

$$
\operatorname{Vol}(A+t B)=\sum_{k=0}^{n}\left(\begin{array}{l}
n \\
k
\end{array}\right) \operatorname{Vol}(A, \ldots, A, B, \ldots, B) t^{k}=: g(t) .
$$

But $A+t B=C+(t+1) B$ and therefore $g(t)=f(t+1)$ for all $t \geq 0$. Therefore $g(t)=f(t+1)$ as polynomials, and $\operatorname{Vol} C=f(0)=g(-1)$. Plugging into 3 gives the desired result.

Having established (2), separate the given Minkowski sum for $P$ into its positive and negative parts as $P=Q-R$, where $Q=x_{1} Q_{1}+\cdots+x_{r} Q_{r}$ and $R=y_{1} R_{1}+\cdots+y_{s} R_{s}$ with $x_{i}, y_{i} \geq 0$. For positive $t$ we can write $Q+t R=\sum x_{i} Q_{i}+\sum t y_{j} R_{j}$, which gives two formulas for $\operatorname{Vol}(Q+t R)$.

$$
\begin{aligned}
\operatorname{Vol}(Q+t R) & =\sum_{k=0}^{n}\left(\begin{array}{l}
n \\
k
\end{array}\right) \operatorname{Vol}(Q, \ldots, Q, R, \ldots, R) t^{k} \\
& =\sum_{\substack{1 \leq i_{a} \leq r \\
1 \leq j_{b} \leq s}} \operatorname{Vol}\left(Q_{i_{1}}, \ldots, Q_{i_{n-k}}, R_{j_{1}}, \ldots, R_{j_{k}}\right) x_{i_{1}} \cdots x_{i_{n-k}} y_{j_{1}} \cdots y_{j_{k}} t^{k}
\end{aligned}
$$

The last two expressions must be equal as polynomials. A priori, we cannot plug $t=-1$ into this equation; but instead, we can use the formula for $\operatorname{Vol}(Q-R)$ from (2), and then plug in coefficient by coefficient. That gives the desired result.

Theorem 3.3 If a connected matroid $M$ has $n$ elements, then the volume of the matroid polytope $P_{M}$ is

$$
\operatorname{Vol} P_{M}=\frac{1}{(n-1) !} \sum_{\left(J_{1}, \ldots, J_{n-1}\right)} \tilde{\beta}\left(M / J_{1}\right) \widetilde{\beta}\left(M / J_{2}\right) \cdots \widetilde{\beta}\left(M / J_{n-1}\right),
$$

summing over the ordered collections of sets $J_{1}, \ldots, J_{n-1} \subseteq[n]$ such that, for any distinct $i_{1}, \ldots, i_{k}$, $\left|J_{i_{1}} \cap \cdots \cap J_{i_{k}}\right|<n-k$.

Proof: Postnikov (14, Corollary 9.4) gave a formula for the volume of a (positive) Minkowski sum of simplices. We would like to apply his formula to the signed Minkowski sum in Theorem 2.5, and Proposition 3.2 makes this possible.

In Theorem 3.3, the hypothesis that $M$ is connected is needed to guarantee that the matroid polytope $P_{M}$ has dimension $n-1$. More generally, if we have $M=M_{1} \oplus \cdots \oplus M_{k}$ then $P_{M}=P_{M_{1}} \times \cdots \times P_{M_{k}}$ so the $((n-k)$-dimensional $)$ volume of $P_{M}$ is $\operatorname{Vol} P_{M}=\operatorname{Vol} P_{M_{1}} \cdots \operatorname{Vol} P_{M_{k}}$.

\section{Independent Set Polytopes}

In this section we show that our analysis of matroid polytopes can be carried out similarly for the independent set polytope $I_{M}$ of a matroid $M$, which is the convex hull of the indicator vectors of the independent sets of $M$. The inequality description of $I_{M}$ is known to be:

$$
I_{M}=\left\{\left(x_{1}, \ldots, x_{n}\right) \in \mathbb{R}^{n}: x_{i} \geq 0 \text { for } i \in[n], \sum_{i \in A} x_{i} \leq r(A) \text { for all } A \subseteq E\right\} .
$$


The independent set polytope of a matroid is not a generalized permutahedron. Instead, it is a $Q$ polytope; i.e., a polytope of the form

$$
Q_{n}\left(\left\{z_{J}\right\}\right)=\left\{\left(t_{1}, \ldots, t_{n}\right) \in \mathbb{R}^{n}: t_{i} \geq 0 \text { for all } i \in[n], \sum_{i \in J} t_{i} \leq z_{J} \text { for all } J \subseteq[n]\right\}
$$

where $z_{J}$ is a non-negative real number for each $J \subseteq[n]$. We can also express these polytopes as signed Minkowski sums of simplices, though the simplices we use are not the $\Delta_{J} \mathrm{~s}$, but those of the form

$$
\begin{aligned}
D_{J} & =\operatorname{conv}\left\{0, e_{i}: i \in J\right\} \\
& =Q_{n}\left(\left\{d(J)_{I}\right\}\right)
\end{aligned}
$$

where $d(J)_{I}=0$ if $I \cap J=\emptyset$ and $d(J)_{I}=1$ otherwise.

The following lemmas on Q-polytopes are proved in a way analogous to the corresponding lemmas for generalized permutahedra as was done in Section 2, and we leave them to the reader.

Lemma 4.1 $Q_{n}\left(\left\{z_{J}\right\}\right)+Q_{n}\left(\left\{z_{J}^{\prime}\right\}\right)=Q_{n}\left(\left\{z_{J}+z_{J}^{\prime}\right\}\right)$

Proposition 4.2 For any $y_{I} \geq 0$ we have

$$
\sum_{I \subseteq[n]} y_{I} D_{I}=Q_{n}\left(\left\{z_{J}\right\}\right)
$$

where $z_{J}=\sum_{I: I \cap J \neq \emptyset} y_{I}$.

Proposition 4.3 Every Q-polytope $Q_{n}\left(\left\{z_{J}\right\}\right)$ can be written uniquel] $]_{\text {(ii) }}^{[a s ~ a ~ s i g n e d ~ M i n k o w s k i ~ s u m ~ o f ~}$ $D_{I} s$ as

$$
Q_{n}\left(\left\{z_{J}\right\}\right)=\sum_{I \subseteq[n]} y_{I} D_{I}
$$

where

$$
y_{J}=-\sum_{I \subseteq J}(-1)^{|J|-|I|} z_{[n]-I}
$$

Proof: We need to invert the relation between the $y_{I} \mathrm{~s}$ and the $z_{J} \mathrm{~s}$ given by $z_{J}=\sum_{I: I \cap J \neq \emptyset} y_{I}$. We rewrite this relation as

$$
z_{[n]}-z_{J}=\sum_{I \subseteq[n]-J} y_{I}
$$

and apply inclusion-exclusion. As in Section 2, we first do this in the case $y_{I} \geq 0$ and then extend it to arbitrary Q-polytopes.

Theorem 4.4 Let $M$ be a matroid of rank $r$ on $E$ and let $I_{M}$ be its independent set polytope. Then

$$
I_{M}=\sum_{A \subseteq E} \widetilde{\beta}(M / A) D_{E-A}
$$

where $\widetilde{\beta}$ denotes the signed beta invariant.

(ii) assuming $y_{\emptyset}=0$ 
The great similarity between Theorems 2.5 and 4.4 is not surprising, since $P_{M}$ is the facet of $I_{M}$ which maximizes the linear function $\sum_{i \in E} x_{i}$, and $\Delta_{I}$ is the facet of $D_{I}$ in that direction as well. In fact we could have first proved Theorem 4.4 and then obtained Theorem 2.5 as a corollary.

Theorem 4.5 If a connected matroid $M$ has $n$ elements, then the volume of the independent set polytope $I_{M}$ is

$$
\operatorname{Vol} I_{M}=\frac{1}{n !} \sum_{\left(J_{1}, \ldots, J_{n}\right)} \widetilde{\beta}\left(M / J_{1}\right) \widetilde{\beta}\left(M / J_{2}\right) \cdots \widetilde{\beta}\left(M / J_{n}\right)
$$

where the sum is over all $n$-tuples $\left(J_{1}, \ldots, J_{n}\right)$ of subsets of $[n]$ such that, for any distinct $i_{1}, \ldots, i_{k}$, we have $\left|J_{i_{1}} \cap \cdots \cap J_{i_{k}}\right| \leq n-k$.

Notice that by Hall's marriage theorem, the condition on the $J_{i} \mathrm{~s}$ is equivalent to requiring that $(E-$ $\left.J_{1}, \ldots, E-J_{n}\right)$ has a system of distinct representatives (SDR); that is, there are $a_{1} \in E-J_{1}, \ldots, a_{n} \in$ $E-J_{n}$ with $a_{i} \neq a_{j}$ for $i \neq j$.

Proof: By Theorem 4.4 and Proposition 3.1 it suffices to compute the mixed volume $\operatorname{Vol}\left(D_{A_{1}}, \ldots, D_{A_{n}}\right)$ for each $n$-tuple $\left(A_{1}, \ldots, A_{n}\right)$ of subsets of $\left.n\right]$. Bernstein's theorem (18) tells us that $\operatorname{Vol}\left(D_{A_{1}}, \ldots, D_{A_{n}}\right)$ is the number of isolated solutions in $(\mathbb{C}-\{0\})^{n}$ of the system of equations:

$$
\left\{\begin{aligned}
\beta_{1,0}+\beta_{1,1} t_{1}+\beta_{1,2} t_{2}+\cdots+\beta_{1, n} t_{n} & =0 \\
\beta_{2,0}+\beta_{2,1} t_{1}+\beta_{2,2} t_{2}+\cdots+\beta_{2, n} t_{n} & =0 \\
& \vdots \\
\beta_{n, 0}+\beta_{n, 1} t+\beta_{n, 2} t_{2}+\cdots+\beta_{n, n} t_{n} & =0
\end{aligned}\right.
$$

where $\beta_{i, 0}$ and $\beta_{i, j}$ are generic complex numbers when $j \in A_{i}$, and $\beta_{i, j}=0$ if $j \notin A_{i}$.

This system of linear equations will have one solution if it is non-singular and no solutions otherwise. Because the $\beta_{i, 0}$ are generic, such a solution will be non-zero if it exists. The system is non-singular when the determinant is non-zero, and by genericity that happens when $\left(A_{1}, \ldots, A_{n}\right)$ has an SDR.

We conclude that $\operatorname{Vol}\left(D_{E-J_{1}}, \ldots, D_{E-J_{n}}\right)$ is 1 if $\left(E-J_{1}, \ldots, E-J_{n}\right)$ has an SDR and 0 otherwise, and the result follows.

Example 4.6 Let $I_{M}$ be the independent set polytope of the uniform matroid $U_{2,3}$. We have $I_{M}=$ $D_{12}+D_{23}+D_{13}-D_{123}$. Theorem 4.5 should confirm that its volume is $\frac{5}{6}$; let us carry out that computation.

The coconnected flats of $M$ are $1,2,3$ and $\emptyset$ and their complements are $\{23,13,12,123\}$. We need to consider the triples of coconnected flats whose complements contain an SDR. Each one of the 24 triples of the form $(a, b, c)$, where $a, b, c \in[3]$ are not all equal, contributes a summand equal to 1 . The 27 permutations of triples of the form $(a, b, \emptyset)$, contribute $a-1$ each. The 9 permutations of triples of the form $(a, \emptyset, \emptyset)$ contribute a 1 each. The triple $(\emptyset, \emptyset, \emptyset)$ contributes a -1 . The volume of $I_{M}$ is then $\frac{1}{6}(24-27+9-1)=\frac{5}{6}$. 


\section{Truncation Flag Matroids}

We will soon see that any flag matroid polytope can also be written as a signed Minkowski sum of simplices $\Delta_{I}$. We now focus on the particularly nice family of truncation flag matroids, introduced by Borovik, Gelfand, Vince, and White, where we obtain an explicit formula for this sum.

The strong order on matroids is defined by saying that two matroids $M$ and $N$ on the same ground set $E$, having respective ranks $r_{M}<r_{N}$, are concordant if their rank functions satisfy that $r_{M}(Y)-r_{M}(X) \leq$ $r_{N}(Y)-r_{N}(X)$ for all $X \subset Y \subseteq E$. (4).

Flag matroids are an important family of Coxeter matroids (4). There are several equivalent ways to define them; in particular they also have an algebro-geometric interpretation. We proceed constructively. Given pairwise concordant matroids $M_{1}, \ldots, M_{m}$ on $E$ of ranks $k_{1}<\cdots<k_{m}$, consider the collection of flags $\left(B_{1}, \ldots, B_{m}\right)$, where $B_{i}$ is a basis of $M_{i}$ and $B_{1} \subset \cdots \subset B_{m}$. Such a collection of flags is called a flag matroid, and $M_{1}, \ldots, M_{m}$ are called the constituents of $\mathcal{F}$.

For each flag $B=\left(B_{1}, \ldots, B_{m}\right)$ in $\mathcal{F}$ let $v_{B}=v_{B_{1}}+\cdots+v_{B_{m}}$, where $v_{\left\{a_{1}, \ldots, a_{i}\right\}}=e_{a_{1}}+\cdots+e_{a_{i}}$. The flag matroid polytope is $P_{\mathcal{F}}=\operatorname{conv}\left\{v_{B}: B \in \mathcal{F}\right\}$.

Theorem 5.1 (4) Cor 1.13.5) If $\mathcal{F}$ is a flag matroid with constituents $M_{1}, \ldots, M_{k}$, then $P_{\mathcal{F}}=P_{M_{1}}+$ $\cdots+P_{M_{k}}$.

As mentioned above, this implies that every flag matroid polytope is a signed Minkowski sum of simplices $\Delta_{I}$; the situation is particularly nice for truncation flag matroids, which we now define.

Let $M$ be a matroid over the ground set $E$ with rank $r$. Define $M_{i}$ to be the rank $i$ truncation of $M$, whose bases are the independent sets of $M$ of rank $i$. One easily checks that the truncations of a matroid are concordant, and this motivates the following definition of Borovik, Gelfand, Vince, and White.

Definition 5.2 (3) The flag $\mathcal{F}(M)$ with constituents $M_{1}, \ldots, M_{r}$ is a flag matroid, called the truncation flag matroid or underlying flag matroid of $M$.

Our next goal is to present the decomposition of a truncation flag matroid polytope as a signed Minkowski sum of simplices. For that purpose, we define the gamma invariant of $M$ to be $\gamma(M)=b_{20}-b_{10}$, where $T_{M}(x, y)=\sum_{i, j} b_{i j} x^{i} y^{j}$ is the Tutte polynomial of $M$.

Proposition 5.3 The gamma invariant of a matroid is given by

$$
\gamma(M)=\sum_{I \subseteq E}(-1)^{r-|I|}\left(\begin{array}{c}
r-r(I)+1 \\
2
\end{array}\right)
$$

Unlike the beta invariant, the gamma invariant is not necessarily nonnegative. In fact its sign is not simply a function of $|E|$ and $r$. For example, $\gamma\left(U_{k, n}\right)=-\left(\begin{array}{c}n-3 \\ k-1\end{array}\right)$, and $\gamma\left(U_{k, n} \oplus C\right)=\left(\begin{array}{c}n-2 \\ k-1\end{array}\right)$ where $C$ denotes a coloop.

As we did with the beta invariant, define the signed gamma invariant of $M$ to be $\widetilde{\gamma}(M)=(-1)^{r(M)} \gamma(M)$.

Theorem 5.4 The truncation flag matroid polytope of $M$ can be expressed as:

$$
P_{\mathcal{F}(M)}=\sum_{I \subseteq E} \widetilde{\gamma}(M / I) \Delta_{E-I}
$$


Proof: By Theorems 2.5 and 5.1, $P_{\mathcal{F}(M)}$ is

$$
\sum_{i=1}^{r} P_{M_{i}}=\sum_{i=1}^{r} \sum_{I \subseteq E} \sum_{J \subseteq I}(-1)^{|I|-|J|}\left(i-r_{i}(E-J)\right) \Delta_{I}
$$

where $r_{i}(A)=\min \{i, r(A)\}$ is the rank function of $M_{i}$. Then

$$
\begin{aligned}
P_{\mathcal{F}(M)} & =\sum_{I \subseteq E}\left[\sum_{J \subseteq I}(-1)^{|I|-|J|} \sum_{i=r(E-J)+1}^{r}(i-r(E-J))\right] \Delta_{I} \\
& =\sum_{I \subseteq E}\left[\sum_{J \subseteq I}(-1)^{|I|-|J|}\left(\begin{array}{c}
r-r(E-J)+1 \\
2
\end{array}\right)\right] \Delta_{I} \\
& =\sum_{I \subseteq E}\left[\sum_{X \subseteq I}(-1)^{|X|}\left(\begin{array}{c}
r_{M /(E-I)}-r_{M /(E-I)}(X)+1 \\
2
\end{array}\right)\right] \Delta_{I} \\
& =\sum_{I \subseteq E} \widetilde{\gamma}(M /(E-I)) \Delta_{I}
\end{aligned}
$$

as desired.

Corollary 5.5 If a connected matroid $M$ has $n$ elements, then

$$
\operatorname{Vol} P_{\mathcal{F}(M)}=\frac{1}{(n-1) !} \sum_{\left(J_{1}, \ldots, J_{n-1}\right)} \widetilde{\gamma}\left(M / J_{1}\right) \widetilde{\gamma}\left(M / J_{2}\right) \cdots \widetilde{\gamma}\left(M / J_{n-1}\right)
$$

summing over the ordered collections of sets $J_{1}, \ldots, J_{n-1} \subseteq[n]$ such that, for any distinct $i_{1}, \ldots, i_{k}$, $\left|J_{i_{1}} \cap \cdots \cap J_{i_{k}}\right|<n-k$.

Proof: This follows from Proposition 3.2 and Theorem 5.4

\section{Acknowledgements}

This work is part of the SFSU-Colombia Combinatorics Initiative. We are very grateful to SFSU and the Universidad de Los Andes for supporting this initiative.

\section{References}

[1] F. Ardila, A. Fink, and F. Rincon. Valuations for matroid polytope subdivisions. Canadian Mathematical Bulletin, to appear.

[2] L. J. Billera, N. Jia, and V. Reiner. A quasysimmetric function for matroids. arXiv:math.CO/0606646. Preprint, 2007. 
[3] A. Borovik, I.M. Gelfand, A. Vince, and N. White. The lattice of flats and its underlying flag matroid polytope. Ann. Combin. 1 (1997) 17-26.

[4] A. Borovik, I.M. Gelfand, and N. White. Coxeter matroids. Birkhäuser, Boston (2003).

[5] H. Crapo. A higher invariant for matroids. J. Combin. Theory 2 (1967), 406-417.

[6] J. A. de Loera, D. Haws, and M. Köppe. Erhart polynomials of matroid polyopes and polymatroids. arXiv:math.CO/0710.4346v1. Preprint, 2007.

[7] H. Derksen. Symmetric and quasisymmetric functions associated to polymatroids. arXiv:math.CO/0801.4393v1 Preprint, 2008.

[8] W. Fulton. Introduction to toric varieties. Ann. of Math. Stud. 131, Princeton Univ. (1993).

[9] I.M. Gelfand, M. Goresky, R.D. MacPherson, and V.V. Serganova. Combinatorial geometries, convex polyhedra, and Schubert cells. Advances Math. 63 (1987), 301-316.

[10] L. Lafforgue. Chirurgie des grassmanniennes. CRM Monograph Series, 19. American Mathematical Society, Providence, RI (2003).

[11] T. Lam and A. Postnikov. Alcoved polytopes. Discrete and Computational Geometry 38 (2007), 453478.

[12] P. McMullen. Valuations and Euler-type relations on certain classes of convex polytopes. Proc. London Math. Soc. 3- 35 (1977), 113-135.

[13] J. G. Oxley. Matroid theory. Oxford University Press. New York, 1992.

[14] A. Postnikov. Permutohedra, associahedra and beyond. arXiv:math.CO/0507163 v1. Preprint, 2005.

[15] D. Speyer. Tropical linear spaces. arXiv:math.CO/0410455. Preprint, 2004.

[16] D. Speyer. A matroid invariant via the K-theory of the Grassmannian. arXiv:math.CO/0603551. Preprint, 2006.

[17] R. Stanley. Eulerian partition of a unit hypercube. Higher combinatorics, M. Aigner ed. Reidel Dordrecht, Boston (1977).

[18] B. Sturmfels. Solving systems of polynomial equations. CBMS Regional Conference in Mathematics, 97. AMS (2002).

[19] D.J.A. Welsh. Matroid theory. Academic Press Inc. London (1976).

[20] N. White. Theory of matroids. Encyclopedia of Mathematics and its Applications, 26. Cambridge University Press (1986). 